\title{
Hogbom's CLEAN algorithm. Impact on astronomy and beyond
}

\author{
T. J. Cornwell
}

\author{
CSIRO Australia Telescope National Facility, PO Box 76, Epping, NSW 1710, Australia \\ e-mail: tim.cornwell@csiro.au
}

Jan Högbom has given the story of his invention of the CLEAN algorithm in a fascinating paper (Högbom 2003) that discusses development of the technique itself rather than the subsequent impact. Here we discuss the historical context of the development of CLEAN, and the impact that it has had throughout science.

At the end of the sixties, radio interferometry was wellestablished as one of the ways to image the radio sky (Thompson et al. 2001). Ryle (Ryle \& Hewish 1960) was soon to win the Nobel prize (along with Hewish - cited for pulsars) for his development of "aperture synthesis". The overall theory - the van Cittert-Zernike theorem and the Fourier transform - was understood and used from day to day in processing observations from radio interferometric telescopes.

The sampling theorem for the Fourier transform required complete sampling of the Fourier plane up to the inevitable cutoff at high spatial frequencies. In practice, this meant that a twelve hour synthesis was necessary for most interferometers if one wanted to image a region of the sky.

All of this was fine, of course, but pragmatic astronomers wanted to go faster - to measure a number of sources in the time that the theory dictated only one object could be imaged. Adding to this was the advent of interferometric telescopes not aligned in the east/west for which the sampling theory was no help. Högbom (2003) has described his use of the Green Bank Interferometer to measure a number of mostly $3 \mathrm{C}$ sources. While he could collect the Fourier coefficients, he had no idea how to make an image from the 50-60 samples obtained for each source. Back in Leiden he evidently tried a number of approaches before settling on the iterative approach that become known as CLEAN.

At first sight, Högbom's algorithm, CLEAN (Högbom 1974, 1983 ) is disconcertingly simple - iteratively identify and remove point sources in the Fourier inverse image (dirty image) using the calculated point spread function to update the dirty image for removing each point source. Since the sky is mostly empty at these resolutions and frequencies, the CLEAN procedure only had to estimate a limited number of source parameters.

Schwarz (1978) provided the first analysis of CLEAN, showing that it could be regarded as a least squares fit of point components to the visibility data. He also showed the conditions under which CLEAN would converge to a zero residual. However, the analysis said nothing about the properties of the CLEAN image obtained for insufficient data.
The answer to this very difficult question has only recently been discovered in the advent of the field of compressive sampling (Candes \& Romberg 2005; Wiaux et al. 2008). Compressive sampling (CS) theory concerns the estimation of sparse objects from incomplete measurements - precisely the domain of the CLEAN algorithm. The principal results from CS are that sparse objects can be recovered from incomplete measurements given some limits on number of objects, number of samples, and incoherence between the object and measurements. If the limits are obeyed, then there are multiple ways to find a solution, including an approach that is equivalent to CLEAN. CS theory is likely to have a substantial impact on radio interferometry, via other more efficient and powerful algorithms, other image representations, and its approach to the quantification of image reliability and robustness.

The other factor in the rise of CLEAN has been the continuing efforts to improve computational speed. The Clark CLEAN took optimal advantage of the FFT and the AP120B array processor to increase the speed of the Högbom CLEAN by an order of magnitude or more (Clark 1980). In the mid eighties, the typical radio astronomy organization would have a VAX 11/780 plus a Floating Point Systems 120B array processor. A substantial fraction of radio synthesis images in the eighties were produced on such machines.

The impact of CLEAN on radio astronomy has been immense. First, there is the accumulated science from the telescopes that have used CLEAN - GBI, MERLIN, WSRT, VLA, VLBI, etc. Observing speed and efficiency have improved enough to allow snapshot imaging, and the dynamic range improvements speak for themselves.

Second, by showing what could be achieved with some postprocessing, CLEAN has encouraged a wave of innovation in synthesis processing that continues to this day. One of the most important secondary products was phase-closure imaging, followed soon thereafter by self-calibration. The first of these allowed VLBI to image the sky accurately, and the second improved the dynamic range of all radio telescopes by one or two orders of magnitude.

Third, there are a substantial number of applications to non-synthesis astronomical measurements, including incomplete time-series analysis (Roberts et al. 1987; Deshpande et al. 1996), RFI excision (Parsons \& Backer 2009), counteracting interstellar broadening of pulses (Bhat et al. 2003), and gravitational lens estimation (Kochanek \& Narayan 1992). 
Outside astronomy, CLEAN has seen a huge range of applications. The simplicity of the algorithm (and of its implementation) and the typically excellent results have encouraged experimentation and adoption in other fields. See, for example, the following examples:

- optical coherence imaging of living tissue (Schmitt 1998);

- synthesis aperture AR sonar imaging (Chick \& Warman 2001);

- characterization of multiple input-multiple output (MIMO) systems; (Poon \& Ho 2003; Yano 2002);

- coherent and incoherent microwave imaging (Tsao \& Steinberg 1988; Bose et al. 2002; Camps et al. 1998);

- tracking and imaging of space debris (Wang et al. 2007);

- data compression (Cohen \& Sandri 1994);

- rinding subsurface objects (e.g. mines) using seismic waves (Alam \& McClellan 2005).

In addition, there are many patents that either refer to CLEAN or draw upon the CLEAN algorithm for some crucial step (see e.g. US Patent 5299577, 5394151, 5862269, 5784492, 6781540, 7215277, 7302269, 11874835).

In summary, the CLEAN algorithm presented in Högbom's paper was ahead of its time. It has been vital in the outburst of radio interferometric imaging in the seventies, eighties, and beyond; enormously generative of new approaches to astronomical data reduction; widely used outside of astronomy; and it plays a role in numerous patents. No wonder it is so highly cited.

\section{References}

Alam, M., \& McClellan, J. 2005, Acoustics, Speech and Signal Processing, Proc. (ICASSP '05), 4, 1097
Bhat, N. D. R., Cordes, J. M., \& Chatterjee, S. 2003, ApJ, 584, 782

Bose, R., Freedman, A., \& Steinberg, B. 2002, IEEE Transactions on Aerospace and Electronic Systems, 38, 89

Camps, A., Bará, J., Torres, F., \& Corbella, I. 1998, Progr. Electromagn. Res, 18, 67

Candes, E. J., \& Romberg, J. K. 2005, in Society of Photo-Optical Instrumentation Engineers (SPIE) Conf. Ser., ed. C. A. Bouman, \& E. L. Miller, 5674, 76

Chick, K., \& Warman, W. 2001, OCEANS, MTS/IEEE Conference and Exhibition, 1, 170

Clark, B. G. 1980, A\&A, 89, 377

Cohen, N., \& Sandri, G. 1994, International Journal of Imaging Systems and Technology, 5, 28

Deshpande, A. A., D’Alessandro, F., \& McCulloch, P. M. 1996, J. A\&A, 17, 7

Högbom, J. A. 1974, A\&AS, 15, 417

Högbom, J. A. 1983, in Indirect Imaging: Proceedings of an International Symposium held in Sydney, Australia, August 30-September 2, ed. C. van Schooneveld (Cambridge University Press)

Högbom, J. A. 2003, in Radio Astronomy at the Fringe, ed. J. A. Zensus, M. H. Cohen, \& E. Ros, ASP Conf. Ser., 300, 17

Kochanek, C. S., \& Narayan, R. 1992, ApJ, 401, 461

Parsons, A. R., \& Backer, D. C. 2009 [arXiv: 0901. 2575]

Poon, A., \& Ho, M. 2003, IEEE Inter. Conf. Commun., 5, 3519

Roberts, D. H., Lehar, J., \& Dreher, J. W. 1987, AJ, 93, 968

Ryle, M., \& Hewish, A. 1960, MNRAS, 120, 220

Schmitt, J. 1998, Journal of Biomedical Optics, 3, 66

Schwarz, U. J. 1978, A\&A, 65, 345

Thompson, A. R., Moran, J. M., \& Swenson, G. W. 2001, Interferometry and synthesis in radio astronomy (A Wiley-Interscience publication)

Tsao, J., \& Steinberg, B. 1988, IEEE Transactions on Antennas and Propagation, 36,543

Wang, Q., Xing, M., Lu, G., \& Boo, Z. 2007, IEEE Transactions on Antennas and Propagation, 55, 3524

Wiaux, Y., Jacques, L., Puy, G., Scaife, A. M. M., \& Vandergheynst, P. 2008 [arXiv: 0812.4933]

Yano, S. 2002, in Vehicular Technology Conference. VTC Spring, IEEE 55th, 3 (IEEE Press), 1200 